\title{
Verständigungshilfen im Dialog mit aphasischen Gesprächspartnern Patienteninformation
}

Menschen mit Aphasie haben Schwierigkeiten beim Sprechen, Verstehen, Lesen und Schreiben. Diese Störungen behindern ihre Teilhabe am Gespräch und am sozialen Leben. Das Denken ist meist nicht oder deutlich geringer betroffen. Aphasiker können auch meist besser kommunizieren als sprechen. Sie wissen, wie man Gespräche führt, wie und wann man z.B. einen Gesprächsschritt übernimmt oder abgibt. Dies gelingt meist auch bei schwerer Aphasie über nonverbale Ausdrucksmittel (Blick, Gestik, Mimik, Körperhaltung) und über hoch automatisierte Redefloskeln.

\section{Welche Sprachschwierigkeiten behindern Menschen mit Aphasie im Gespräch?}

Menschen mit Aphasie haben in unterschiedlichem Ausmaß Schwierigkeiten, ihre Gedanken, Absichten und Wünsche sprachlich zu äußern. Sie suchen nach Wörtern und Formulierungen. Oft verwechseln sie Sprachlaute oder haben Mühe, deutlich zu sprechen. Auch das genaue Verstehen der Sprache ist beeinträchtigt und besonders erschwert in Gruppengesprächen. Im schwersten Fall können aphasische Personen nur noch einzelne Wörter im engen Situationszusammenhang verstehen. Das Wissen über Situation, Handlungen, Menschen, Gewohnheiten, eigene Erfahrungen etc. ist aber in der Regel gut erhalten. So kann es sein, dass beim Besuch im Krankenhaus der Satz „Soll ich Dir die Zeitung bringen?“ gut verstanden wird, wenn der gesunde Gesprächspartner dabei auf die Zeitung blickt oder zeigt und

\section{Info und Beratung}

Bundesverband für die Rehabilitation der Aphasiker e.V. (BRA) www.aphasiker.de, info@aphasiker.de

Weiterführende Literatur: Auer P, Bauer A. Aphasie im Alltag. Stuttgart: Thieme; 2009 anschließend den aphasischen Partner fragend anschaut. Derselbe Satz kann aber am Telefon nicht verstanden werden, weil die Körpersprache fehlt.

\section{Was tun, wenn aphasische Gesprächspartner Sprachver- ständnisprobleme haben? $\nabla$}

Am fragenden Gesichtsausdruck und an begleitenden Handbewegungen können Sie meist erkennen, dass der aphasische Partner sie nicht (ganz) verstanden hat. Welche Verständigungsstrategien können dann hilfreich sein?

- Versuchen Sie, Ihre Äußerung vereinfachend umzuformulieren.

- Verwenden Sie zusätzlich zur Sprache unterstützende Gestik und Mimik.

- Achten Sie darauf, dass Ihre Sätze kurz sind.

- Verwenden sie keine seltenen, schwierigen Wörter.

- Sprechen Sie deutlich und mit guter Betonung.

- Reduzieren Sie insgesamt das Tempo des Gesprächs

- Achten Sie darauf, dass der Aphasiker Ihre Mundbewegungen sehen kann.

Was tun, wenn der Gesprächspartner sich nicht sprachlich äußern kann oder frustriert seine Formulierungsversuche abbricht? $\nabla$

Versuchen Sie mit dem Aphasiker ins Gespräch zu kommen. Bieten Sie Anknüpfungsmöglichkeiten durch Fragen, die sich mit ,ja“ oder „nein“ beantworten lassen. Ermutigen Sie den aphasischen Partner, alle verfügbaren Ausdrucksmittel zu verwenden. Dazu gehören passende Gesten, Zeichnen, aber auch Schreib- und Sprechversuche, die Sie deuten können (z.B. Vielleicht kannst Du mir zeigen, was du sagen willst. Fällt dir ein Wort ein? Dann kann ich raten, was du meinst.).

Aphasien werden häufig durch einen Schlaganfalls ausgelöst. Bitte haben Sie Verständnis dafür, dass durch dieses ein- schneidende Krankheitsereignis nicht nur die Sprache beeinträchtigt sein kann, sondern eventuell auch die Emotionalität. Dies bedeutet, dass der aphasische Patient möglicherweise anders (z.B. schneller frustriert oder gereizt) sein kann. Hier ist vom Gesprächspartner dann auch mehr Geduld gefragt.

\section{Was tun im Gespräch mit Dritten? $\nabla$}

Ermöglichen Sie dem Aphasiker die Teilhabe an der Kommunikation mit Dritten. Für weniger vertraute Gesprächspartner kann die aphasische Sprache wie eine „Fremdsprache“ klingen. Übernehmen Sie dann die Rolle des Übersetzers. Selbst Menschen mit schwerer Aphasie können an Gesprächen teilnehmen, wenn Sie als vertrauter Partner die gestischen Mitteilungen und aphasischen Sprachäußerungen für Dritte übersetzen oder ergänzen.

\section{Was ist darüber hinaus wichtig? $\nabla$}

Das Gespräch dient nicht nur der Mitteilung von Informationen, sondern auch zur Gestaltung von sozialen Beziehungen und Rollen. Sprachstörungen erschweren deshalb nicht nur die Informationsvermittlung.

Korrigieren Sie den aphasischen Partner im Gespräch mit anderen Personen nur mit dessen Zustimmung, um ihm einen Gesichtsverlust zu ersparen. Nur wenn Sie die „Erlaubnis“ des Betroffenen über seinen Blick oder eine Geste erhalten, sollten sie unterstützend eingreifen.

Im Alltagsgespräch sollen sie das Sprachrohr des Aphasikers sein und nicht der Lehrer oder Therapeut.

\section{Dr. phil. Luise Springer, Aachen}

\section{überreicht durch Thieme und}

